Document downloaded from:

http://hdl.handle.net/10251/83626

This paper must be cited as:

Guijarro, L.; Pla, V.; Vidal Catalá, JR.; Naldi, M. (2016). Maximum-Profit Two-Sided Pricing in Service Platforms Based on Wireless Sensor Networks. IEEE Wireless Communications Letters. 5(1):8-11. doi:10.1109/LWC.2015.2487259.

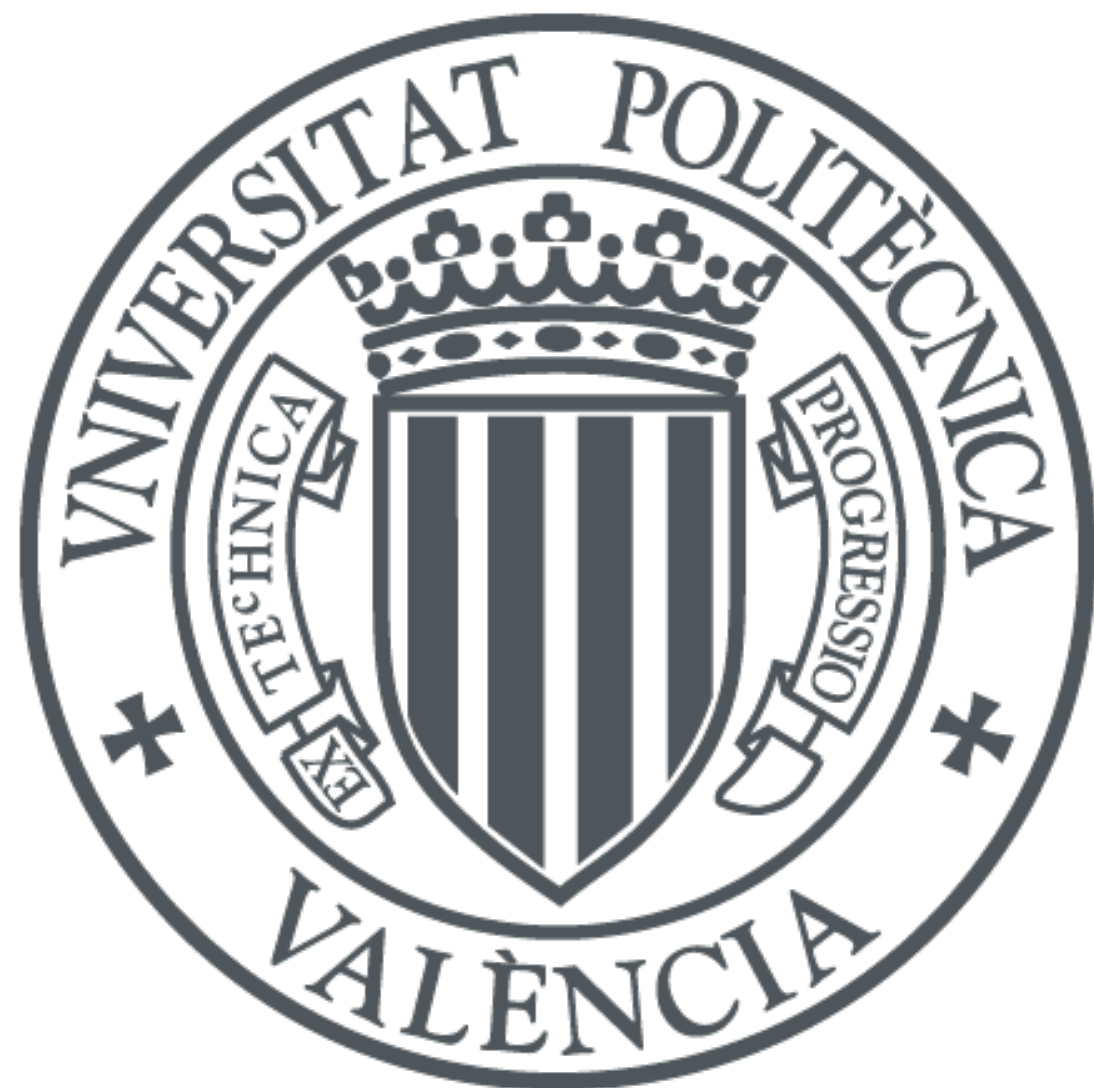

The final publication is available at

http://dx.doi.org/10.1109/LWC.2015.2487259

Copyright Institute of Electrical and Electronics Engineers (IEEE)

Additional Information

(c) 2015 IEEE. Personal use of this material is permitted. Permission from IEEE must be obtained for all other users, including reprinting/ republishing this material for advertising or promotional purposes, creating new collective works for resale or redistribution to servers or lists, or reuse of any copyrighted components of this work in other works. 


\title{
Maximum-profit two-sided pricing in service platforms based on Wireless Sensor Networks
}

\author{
Luis Guijarro, Vicent Pla, Jose R. Vidal, and Maurizio Naldi
}

\begin{abstract}
A business model for Internet-of-Things-based services is proposed whereby a platform serves as an intermediary between human users and Wireless Sensor Networks (WSNs). The platform, acting as a monopolist, posts both the price paid by each user and the price paid to each WSN so as to maximize its profits. In this setting, we propose, analyze and compare two alternative payment schemes for the WSN side. We demonstrate that the two payment schemes are equivalent from every stakeholders point of view. And then we show that there is a user cost ceiling, which depends both on the number of WSNs and the strength of the cross externality that the WSNs creates on the users, below which the take-up is maximum.
\end{abstract}

Index Terms-Wireless sensor networks, two-sided markets, service provision.

\section{INTRODUCTION}

The "Internet of Things" (IoT) is one of the hottest topics being debated today across industries worldwide. The estimates of the number of smart objects in homes, offices, factories, vehicles and elsewhere are 50 billion by 2020, up from 12.5 billion in 2010 [1]. Although smart objects are becoming omnipresent, the fact is that the market for services related to these objects is immature.

This paper aims to contribute to the understanding of a sustainable business model for wireless-sensor-network-based services, which is a likely scenario for the IoT. Specifically, this paper proposes a business model built around a platform which distributes the sensing information to the relevant parties and takes care of bundling the solutions, setting the tariffs, billing the customers and providing customer care [1].

In the analysis of this platform-based business model for IoT, we borrow the concept of two-sided markets, as presented by [2] and as analyzed by [3]. Some specifics related to the WSN's operation are incorporated in the model, such as the influence of the sensing rate over the user utility, and the variable cost dependence on the sensing rate. We investigate how the pricing schemes that a platform applies to each side of the IoT service (users and wireless sensor networks) may increase the total service take up at each side [4].

As far as the authors are aware, there are some reports that discuss which requirements a sustainable business model should comply in an Internet of Things scenario [5] [6], but there is no paper that approaches this issue formally as our manuscript.

This work has been supported by the Spanish Ministry of Economy and Competitiveness through project TIN2013-47272-C2-1-R.

L. Guijarro, V. Pla, and J. R. Vidal are with ITACA, Universitat Politècnica de València, 46022Spain (e-mail: \{lguijar, vpla, jrvidal\}@dcom.upv.es)

M. Naldi is with Universit'a di Roma Tor Vergata, Italy (e-mail: naldi@ disp.uniroma2.it)
The structure of the paper is as follows. Next section describes the business model and the two payment methods, and the basis of the analysis is described. Section III presents the main results of the paper and Section IV draws some conclusions and points to future lines of work.

\section{MODEL}

The scenario modeled in this paper comprises $N$ Wireless Sensor Infrastructure Providers (WSIP), one service provider, and $M$ users. Two models are presented in this section, where the difference lays in the payment made by the service provider to each WSIP . A monopolistic service provider is then assumed in the paper. We acknowledge that no barrier can be identified in this market, so that more realistic scenarios where several service providers compete against each other should be modeled. However, at the current stage, the study of a monopolistic model can be regarded as representative and provide valuable insights to approach the study of more complex scenarios.

\section{A. Lump-sum payment model}

This is the baseline model, hereafter also referred as model A, where the service provider pays a lump sum to each WSIP. The model is depicted in Fig. 1.

Each WSIP operates and manages a Wireless Sensor Network (WSN). The WSN island senses information which is bundled by the service provider in order to compose useful services to the users.

WSN $\mathrm{j}$ is able to sense at a rate $r_{j}$. This rate not only influences the user utility, as stated below, but also contributes to the costs incurred. Specifically, we model WSIP j's costs as proportional to its sensing rate, i.e. $f \cdot r_{j}$, modeling the fact that the more a WSN senses, the more resources it consumes, e.g., battery. We model the heterogeneity in terms of $r_{j}$ through a random variable $\mathcal{R}$ uniformly distributed in the interval $[0,1]$. Additionally, the service provider pays a lump-sum fee $q$ to each WSIP. Although more sophisticated payments may be modeled at this stage-e.g., differential pricing-, a simple payment is desirable so as to remain focused on the effects that the alternative payment presented in Section II-B may introduce in the system.

Therefore, provided that WSIP $j$ joins the service provider platform, it will get the profits

$$
\Pi_{j}^{A}=q-f r_{j} .
$$

Otherwise, the WSIP will get zero revenues and profits. The number of WSIPs that join the platform is denoted by $n$. 


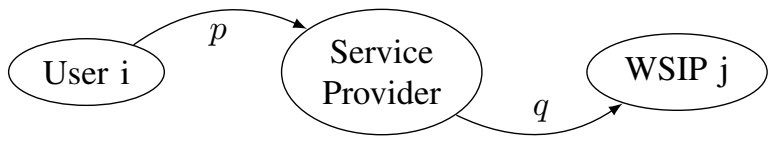

Fig. 1. Lump-sum payment model

Users are interested in accessing a range of services that the service provider composes from the WSN islands operated by the WSIPs.

Each user has a utility that comprises both objective aspects and unobserved aspects. The unobserved aspects may reflect subjective features of the service consumption, and these aspects are responsible for the heterogeneity of the user consumption behavior. We propose to model these aspects with a uniform random variable that reduces the objective part of the utility in a linear manner. More specifically, each user has a type denoted by $x_{i}$, which is modeled as a uniformly distributed random variable $\mathcal{X}=U[0,1]$, and a disutility equal to $t$ times $x_{i}$ reduces the objective part of the utility ${ }^{1}$.

As regards the objective aspects, sensor nodes produce utility by sensing and reporting data to the WSIP and ultimately, to the service provider. Therefore, following [7], the utility that the users get from the WSN-based services is assumed to depend on the aggregate sensing rate $\sum_{j=1}^{n} r_{j}$, through a positive, non-decreasing and concave function $\Phi(\cdot)$.

Therefore, provided that user $i$ subscribes for the service, his/her utility is specified by

$$
u_{i}=v+\Phi\left(\sum_{j=1}^{n} r_{j}\right)-t x_{i}-p,
$$

where $v$ is the net value that a user receives from accessing the platform irrespective of the amount of service received, accounting also for network access fees; and $p$ is the lump-sum payment for the service. From (2), it follows that there always exists an cross externality from the number of WSIPs to the number of users; that is, the more WSIPs join the platform, the greater utility the users get. We assume that a user will get zero utility if he/she chooses not to subscribe for the service.

The service provider performs two basic roles in the model: it composes services which are based on the information sensed by the different WSN islands; and it acts as an intermediary between users and WSIPs, which allows to decouple the pricing schemes on each side.

The profits of the service provider is given by the revenues from the users $(p m)$ minus the cost incurred in paying the WSIPs $(q n)$ :

$$
\Pi_{p}^{A}=p m-q n
$$

Analysis: As hinted above, we will see that the demand for services depends on the expected number of WSIPs connected, since the more WSIPs are aggregated by the service provider, the more users will subscribe for service.

\footnotetext{
${ }^{1}$ This approach can be also interpreted as an generalization of the Hotelling model, where $x_{i}$ is the user location, the service provider is located at $x=0$, and $t x_{i}$ is the transportation cost.
}

We assume, first, that the number of WSIPs that join the platform is $n^{e}$ and that the rate from each of these WSIPs is $r_{j}^{e}, j=1, \ldots, n^{e}$.

User $i$ will subscribe to the service if $u_{i} \geq 0$, which corresponds to the random event

$$
\mathcal{X} \leq \frac{1}{t}\left(v+\Phi\left(\sum_{j=1}^{n^{e}} r_{j}^{e}\right)-p\right)
$$

This event has a probability equal to

$$
\Psi\left(\frac{1}{t}\left(v+\Phi\left(\sum_{j=1}^{n^{e}} r_{j}^{e}\right)-p\right)\right),
$$

where $\Psi$ is defined as follows:

$$
\Psi(u)= \begin{cases}0 & \text { if } u<0 \\ u & \text { if } 0 \leq u<1 \\ 1 & \text { if } 1 \leq u\end{cases}
$$

The number of subscribers $\mathcal{M}$ is then a random variable, specifically, a binomial random variable with parameters $M$ and (5). Therefore, the expected number of subscribers, conditioned to the values $n^{e}, r_{1}^{e}, r_{2}^{e}, \cdots, r_{n}^{e}$, is equal to

$$
\begin{aligned}
m & \triangleq E\left[\mathcal{M} \mid n^{e}, r_{1}^{e}, r_{2}^{e}, \cdots, r_{n^{e}}^{e}\right] \\
& =M \Psi\left(\frac{1}{t}\left(v+\Phi\left(\sum_{j=1}^{n^{e}} r_{j}^{e}\right)-p\right)\right) .
\end{aligned}
$$

We proceed now in a similar way for the number of connected WSIPs. WSIP $j$ will join the platform if $\Pi_{j}^{A} \geq 0$, which corresponds to a random event with probability

$$
P(q-f \mathcal{R} \geq 0)=P(\mathcal{R} \leq q / f)=\Psi(q / f) .
$$

Following a similar reasoning as with $m$, the expected number of WSIPs is equal to

$$
n \triangleq E[\mathcal{N}]=N \Psi(q / f) .
$$

Additionally, the rate $\mathcal{R}$ of a connected WSIP has an expected value equal to

$$
r \triangleq E[\mathcal{R} \mid \mathcal{R} \leq q / f]=\frac{1}{2} \Psi(q / f)
$$

We now look for an equilibrium where each side's assumptions are fulfilled, i.e. $n^{e}=n, m^{e}=m$, and $\sum_{j=1}^{n^{e}} r_{j}^{e}=n \cdot r$, so that, Equation (7) becomes

$$
m=M \Psi((v+\Phi(n r)-p) / t)
$$

Finally, from (10), (9) and (11), we can compute the expected WSIP and service provider profits as functions of $p$ and $q$. Assuming that the monopoly platform is free to set both subscription price $p$ to the users and fee $q$ to the providers, the platform faces the problem of choosing $p$ and $q$ to maximize $\Pi_{p}$. Thus, an maximization problem should be solved, as it is described below in Section III. 


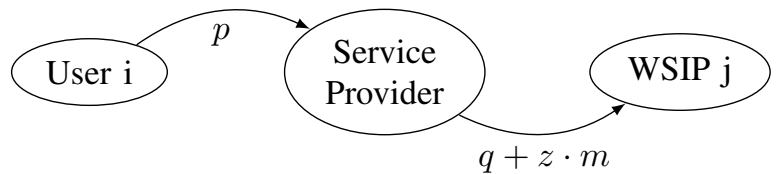

Fig. 2. Per-subscriber payment model

\section{B. Per-subscriber payment model}

In this model, hereafter also referred as model B, we propose that the service provider pays an additional amount $z$ per subscriber to each connected WSIP, i.e. the total payment is now $q+z m$, where $m$ is the number of users that subscribe to the service ${ }^{2}$. The payment flow is shown in Fig. 2. This payment is intended to create an additional incentive for the WSIPs to join the platform, since they will be rewarded collectively as more users subscribe. We already know that the number of subscribers depends on the number of WSIPs that join the platform. This way, a closed-loop feedback may be created that increases both the platform profits and the service take up.

The utility that a subscriber gets from the service provider is still given by (2). However, WSIP's profits and the service provider profits are now given by

$$
\begin{aligned}
& \Pi_{j}^{B}=q+z m-f r_{j}, \\
& \Pi_{p}^{B}=p m-(q+z m) n .
\end{aligned}
$$

Eq. (12) shows that there exists now a cross externality from the users' side to the WSIPs' side which was absent in the previous model — see (1) — and which is introduced by the proposed payment. This cross externality supplements the one present in the user utility-see (2). A bidirectional cross externality is then generated. This may recreate a two-sided market where the service provider acts as a platform. If so, the service provider would internalize the bidirectional cross externality, improve its profits and increase the take-up of either the users or the WSIPs, or both.

Analysis: Let us assume that the number of subscribers is $m^{e}$. WSIP $j$ will join the platform if $\Pi_{j}^{B} \geq 0$, which corresponds to a random event with probability

$$
P\left(q+z m^{e}-f \mathcal{R} \geq 0\right)=\Psi\left(\left(q+z m^{e}\right) / f\right) .
$$

Following a similar reasoning as in previous section, the following values result:

$$
\begin{aligned}
m & =M \Psi((v+\Phi(n r)-p) / t), \\
n & =N \Psi((q+z m) / f), \\
r & =1 / 2 \Psi((q+z m) / f)=n / 2 N .
\end{aligned}
$$

The expressions for $m$ and $n$ are obtained by solving the system of equations (15) and (16), which is more involved that the direct substitution of (9) into (11) in model A.

A profit maximization problem needs again to be solved, as it is described below.

\footnotetext{
${ }^{2}$ The proposed payment is different from a two-part tariff. While the proposed payment depends on the opposite side to the side where it is applied, a two-part tariff does not.
}

\section{RESULTS}

In this section, we discuss the numerical results for the two models analyzed in the previous section.

A squared root function has been chosen for $\Phi$, following the examples considered in [7], i.e., $\Phi(n r)=b \sqrt{n r}=$ $b n / \sqrt{2 N}$.

The profit maximization problems A and B have been first solved analytically. Since $\Psi$ is a piecewise linear function defined in three different intervals, solving (15) and (16) or either, trivially, (11) and (9)-yields nine solution types for $(m, n): m=n=0 ; m=0,0<n<N ; m=0$, $n=N ; 0<m<M, n=0 ; 0<m<M, 0<n<N$; $0<m<M, n=M ; m=M, n=0 ; m=M, 0<n<N$; and $m=M, n=M$. Each solution has a feasibility region in the $p q$-plane that is delimited by a piecewise linear curve, and by substituting the solution into (13) —or either (3)—, in each region we have a function $\Pi_{p}(p, q)$ that is a polynomial of degree 1 or 2 . To solve the maximization problem, we first solve a constrained maximization problem in each of the nine regions, and then compare the obtained maxima among them to obtain the global maximum. We would like to stress that both maximization problems have been approached using a similar methodology, but the specifics of the solving have been different, which cannot be shown here due to the page limit. For the detailed derivation of expressions, the reader is referred to the technical report that accompanies this paper [8].

The problems have also been solved numerically, where it was not possible to use any gradient-based algorithm, because in both models the function to optimize is not smooth. Furthermore, the maximization problem in model B is non-linear. For this reason we have used a gradient-free algorithm, which has a much more robust behavior under such problems. We have chosen the modification of the cuckoo search algorithm described in [9].

\section{A. Payment equivalence}

The proposed payment $q+z m$ in model B aimed to generate a cross externality that complemented the one that the number of connected WSIPs had created on the subscribers' utility.

Both analytical and numerical results have been obtained for the two payments, and it can be shown that both are equivalent from every stakeholder's point of view, that is, the service provider's and WSIP's profits, the number of subscribers, and the number of connected WSIPs. The price paid by the users is identical in both payments; as regards the fee paid to the WSIPs, the results show that $q^{A}=q^{B}+m z$, that is, the platform adjusts $q^{B}$ to produce an overall payment to each WSIP that is equal to the fee $q^{A}$.

\section{B. Optimum analysis}

The following figures show the optimum price $p$ and fees $q^{A}$ and $q^{B}$ (Fig.3), the corresponding values $m$ and $n$ (Fig. 4), and the maximum $\Pi_{p}$ and corresponding $\Pi_{w}$-which is the mean value of the profit of a connected WSIP (Fig. 5). All values are represented as functions of parameter $t^{3}$.

\footnotetext{
${ }^{3}$ The plots have been generated with parameter values $v=1, b=1$, $f=1, z=0.1, M=40$ and $N=30$. Furthermore, $B>1$. If $B \leq 1$, the plots would not show the region $(C, B \cdot C]$.
} 


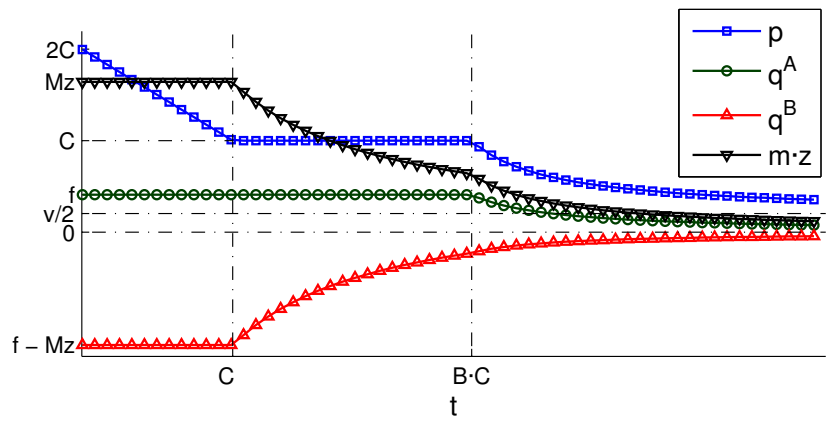

Fig. 3. Subscription price and payments as a function of $t$

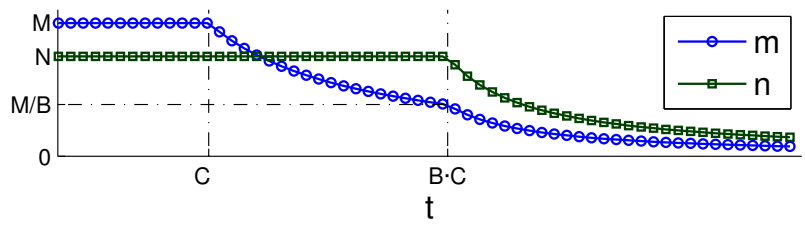

Fig. 4. Number of subscribers and of WSIPs as a function of $t$

We can state the following facts:

- As stated above, although $q^{A}$ and $q^{B}$ are different, they comply with $q^{A}=q^{B}+m z$ (Fig. 3).

- Provided that $t \leq C$, where $C \triangleq \frac{1}{2}(v+b \sqrt{N / 2})$, i.e., that the user costs are small compared with a quantity that increases with the number of WSIPs $N$ and the strength of the cross externality $b$,

- in the optimum, all users subscribe $(m=M)$ and all WSIPs connect $(n=N)$ (Fig. 4);

- as $t$ increases, which means higher costs born by the users, the platform chooses a lower $p$ in order to compensate for the increase in $t$ (Fig. 3), and it succeeds in keeping $m=M$ (Fig. 4), but $\Pi_{p}$ decreases (Fig. 5).

- When $C \leq t \leq B \cdot C$, where $B \triangleq \frac{M b}{f \sqrt{8 N}}$,

- the platform can no longer avoid that $m$ decreases (Fig. 4), so that it has no incentive in lowering $p$ (Fig. 3);

- $q^{A}$ and $q^{B}$ are set to a value that keeps all WSIPs connected ( $n=N$, Fig. 4) and its profits $\Pi_{w}$

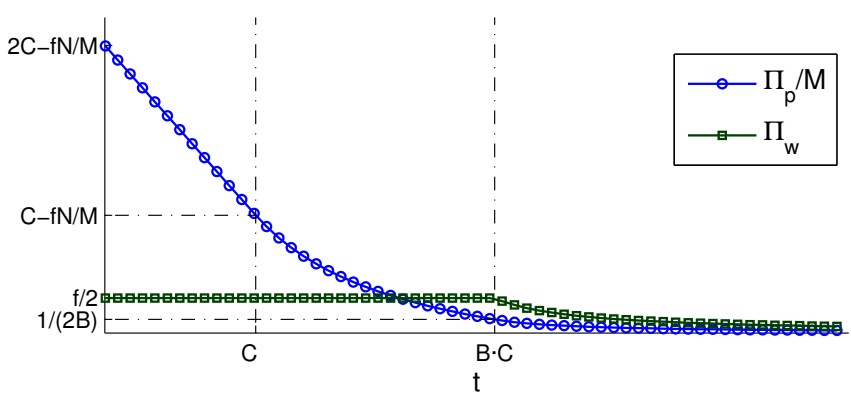

Fig. 5. Service provider's profits and WSIP's profits as a function of $t$ unaltered (Fig. 5);

- the decrease in $m$ causes that service provider's profit $\Pi_{p}$ decreases (Fig. 5).

- As $t$ increases beyond $B \cdot C$, i.e., a quantity which increases almost linearly with the number of users $M$ and $b$,

- the platform chooses a lower price $p$ and a lower $q$ to try to compensate for the increase in $t$ (Fig. 3), but it cannot avoid that both $m$ and $n$ decrease (Fig. 4);

- the decrease in $q^{A}$ and in $q^{B}+m z$ causes that $\Pi_{w}$ decreases (Fig. 5);

- the decrease in $m$ and in $n$ causes that $\Pi_{p}$ decreases (Fig. 5).

The above facts show that there is a user cost ceiling $C$ modulated by $B$, under some conditions, below which the take-ups $m$ and $n$ are maximum. Beyond this cost ceiling, the take-ups decrease. Note that high values for $\mathrm{C}$ can be achieved in scenarios with a high availability of WSIPs and with a strong externality $b$.

\section{CONCLUSIONS}

A business model is analyzed for a service platform that intermediates between WSNs and users. Two payment methods have been proposed and analyzed through solving the respective profit maximization problems, and the equivalence between the two methods have been stated. The behavior of the model for a typical parameter setting has been described.

We argue that the equivalence between the two payment methods is an intrinsic characteristic of the business model. Specifically, we argue that the payment flows to and from the WSIPs makes the system insensitive to a change in the payment method.

\section{REFERENCES}

[1] D. Evans, "The internet of things. how the next evolution of the internet is changing everything," White paper, vol. 2011, no. 4, pp. 1-11, 2017.

[2] M. Armstrong, "Competition in two-sided markets," The RAND Journal of Economics, vol. 37, no. 3, pp. 668-691, 2006.

[3] N. Economides and J. Tåg, "Network neutrality on the internet: A twosided market analysis," Information Economics and Policy, vol. 24, no. 2, pp. 91-104, 2012.

[4] J.-M. Bohli, C. Sorge, and D. Westhoff, "Initial observations on economics, pricing, and penetration of the internet of things market," $A C M$ SIGCOMM Computer Communication Review, vol. 39, no. 2, pp. 50-55, 2009.

[5] E. Fleisch, M. Weinberger, and F. Wortmann, "Business models and the internet of things," in Interoperability and Open-Source Solutions for the Internet of Things. Springer, 2015, pp. 6-10.

[6] T. Keskin and D. Kennedy, "Strategies in smart service systems enabled multi-sided markets: Business models for the internet of things," in System Sciences (HICSS), 2015 48th Hawaii International Conference on. IEEE, 2015, pp. 1443-1452.

[7] B. Zhang, R. Simon, and H. Aydin, "Maximum utility rate allocation for energy harvesting wireless sensor networks," in Proceedings of the 14th ACM international conference on Modeling, analysis and simulation of wireless and mobile systems. ACM, 2011, pp. 7-16.

[8] L. Guijarro, V. Pla, J. R. Vidal, and M. Naldi, "Maximumprofit two-sided pricing in service platforms based on wireless sensor networks," no. TELPOL95h, 2015. [Online]. Available: http: //personales.upv.es/lguijar/pubs/TELPOL95h.pdf

[9] S. Walton, O. Hassan, K. Morgan, and M. Brown, "Modified cuckoo search: A new gradient free optimisation algorithm," Chaos, Solitons \& Fractals, vol. 44, no. 9, pp. $710-718,2011$. [Online]. Available: http://www.sciencedirect.com/science/article/pii/S096007791100107X 